\title{
Kinetics of hydrocortisone sodium phosphate penetration into the human aqueous humor after topical application
}

\author{
Carlo Cagini ${ }^{1}$, Alessio Muzi $^{1}$, Greta Castellucci ${ }^{1}$, Giulia Ragna ${ }^{1}$, Marco Lupidi ${ }^{1}$, Husam \\ BR Alabed $^{1}$, and Roberto Maria Pellegrino ${ }^{1}$ \\ ${ }^{1}$ University of Perugia
}

August 10, 2021

\begin{abstract}
Aim of the study: Hydrocortisone is a soft steroid with low anti-inflammatory properties and a short duration of action, used to manage several ocular conditions. The clinical benefits and side effects associated with hydrocortisone are well documented, but its basic pharmacokinetic in the eye is yet to be fully elucidated. The purpose of this study is to investigate the anterior chamber penetration capabilities of hydrocortisone when used in different concentrations as eye drops treatment. Materials and Methods: This is a double-blind, single-center, randomized clinical trial performed at the Department of Medicine and Surgery of the University of Perugia (Italy) on consecutive patients who have undergone phacoemulsification with intraocular lens implantation. Patients were randomly assigned on the morning of surgery to receive a single instillation of $0.33 \%$ (group A) or $0.001 \%$ (group B) hydrocortisone sodium phosphate solution. Group of patients C did not receive any treatment and was used to measure the hydrocortisone endogenous levels. Before surgery, one aliquot of aqueous humor for each patient was aspirated. The time of collection for each sample was recorded. Hydrocortisone concentrations were then stratified into six interval classes of 30 minutes each. Results: The mean concentration of hydrocortisone was significantly higher in group A $(25.2 \pm 12.4 \mathrm{ng} / \mathrm{ml})$ compared with group B $(7.11 \pm 1.51 \mathrm{ng} / \mathrm{ml})$ and compared to the mean hydrocortisone endogenous levels $(3.92 \pm 1.18 \mathrm{ng} / \mathrm{ml})(\mathrm{p}<0.0001)$. No statistically significant differences of hydrocortisone mean concentrations between group B and the mean endogenous levels were found. Conclusions: Considering the frequent need for prolonged topical steroid therapies and the possible consequent undesirable side effects, ophthalmologists should consider the lowest clinically effective dose of hydrocortisone useful to obtain the desired therapeutic effect and in an adequate time, to minimize the amount of steroids into the anterior chamber and to avoid side effects, such as intraocular pressure increase or cataract development.
\end{abstract}

Article type : Short Report

Kinetics of hydrocortisone sodium phosphate penetration into the human aqueous humor after topical application

Running header: Kinetics of hydrocortisone

Carlo Cagini ${ }^{1}$, Alessio Muzi ${ }^{1}$, Greta Castellucci ${ }^{1}$, Giulia Ragna ${ }^{1}$, Marco Lupidi ${ }^{1}$, Husam BR Alabed ${ }^{2}$, Roberto Maria Pellegrino ${ }^{2}$

${ }^{1}$ Department of Medicine and Surgery, University of Perugia, Perugia, Italy

${ }^{2}$ Department of Chemistry, Biology and Biotechnology, University of Perugia, Perugia, Italy

Corresponding author:

Prof. Carlo Cagini, M.D.: carlo.cagini@unipg.it

ORCID: 0000-0002-3812-9219 
Department of Medicine and Surgery, University of Perugia, S. Maria della Misericordia Hospital, Piazza Menghini 1, S. Andrea delle Fratte, 06156 Perugia, Italy

\section{Ethics approval and informed consent}

The study protocol was approved by the local ethics committee (CER Umbria, register number 3500/19). Written informed consent was obtained from all patients.

\section{Consent for publication}

Not required as this manuscript does not include details, images or videos related to the participants.

\section{Data availability}

Data may be made available upon reasonable request.

\section{Funding}

No funding to declare.

\section{Disclosures}

No conflict of interest to declare.

\section{Authors' contributions}

Study design: Carlo Cagini, Alessio Muzi, Husam BR Alabed, Roberto Maria Pellegrino. Data collection and interpretation: All. Manuscript writing: Alessio Muzi, Giulia Ragna, Husam BR Alabed. Manuscript editing and approval to submit: all.

\section{Acknowledgments}

Editorial assistance was provided by Simonetta Papa, PhD, and Aashni Shah (Polistudium SRL, Milan, Italy). This assistance was supported by Alfa Intes.

\section{Hosted file}

Lavoro PK idrocortisone_AS_151721.docx available at https://authorea.com/users/429939/ articles/533518-kinetics-of-hydrocortisone-sodium-phosphate-penetration-into-the-humanaqueous-humor-after-topical-application 
figures/Figure-1/Figure-1-eps-converted-to.pdf 